\title{
SEGURANÇA DO PACIENTE: PREVENÇÃO DE ÚLCERA POR PRESSÃO EM UNIDADE DE TERAPIA INTENSIVA
}

\section{Patient Safety: Pressure ulcer prevention in intensive care unit}

Mariane Onofre Pereira ${ }^{1}$, Sabrina Cardoso Ludvich ${ }^{1}$, Jaqueline Aparecida Erig Omizzolo $^{1}$

1 Universidade do Planalto Catarinense - UNIPLAC

\section{Endereço para correspondência:}

Jaqueline Aparecida Erig Omizzolo

Endereço: Av. Dom Daniel Hostin, 474, Bairro Frei Rogério - Lages/SC

Cep: 88508-200

Email: jacky-erig@hotmail.com 


\title{
Resumo
}

Este estudo teve como objetivo conhecer as ações assistenciais desenvolvidas pela equipe de enfermagem de uma unidade de terapia intensiva de um hospital de referência da serra catarinense, em relação a úlceras por pressão. Trata-se de um artigo original, Trabalho de Curso no tema de segurança do paciente. É um estudo de caráter descritivo e de abordagem qualitativa, desenvolvido através de Pesquisa Convergente Assistencial. Participaram do estudo 12 sujeitos e como resultados levantaram-se três categorias de análise: conhecimento profissional; classificação de risco dos pacientes; notificação das UPPs. Dentre as ações assistenciais na prevenção de úlcera por pressão destaca-se como principal a mudança de decúbito. $\mathrm{Na}$ classificação de risco dos pacientes utilizou-se a escala de Braden. Com este estudo evidenciou-se que os dados encontrados assemelham-se à realidade descrita nos estudos existentes. A alta prevalência e incidência de úlcera por pressão na amostra analisada reforça o problema. Sugere-se a utilização da Escala de Braden como instrumento assistencial para a prevenção deste tipo de evento adverso evitável. Palavras-chave: Úlceras por pressão; Unidade de Terapia Intensiva; Segurança do Paciente; Enfermagem.

\begin{abstract}
This study aimed to know the care actions developed by the nursing staff of intensive care unit of a referral hospital of Santa Catarina saw, in relation to pressure ulcers. This is an original article, course work in patient safety issue. It is a study of descriptive and qualitative approach, developed by Care Convergent Research. The study enrolled 12 subjects and as a result bring up three categories of analysis: professional knowledge; risk classification of patients; notification of UPP. Among the care actions to prevent pressure ulcer stands out as the main position change. In patients risk classification, we used the Braden Scale. This study showed that the data found resemble the reality described in the existing studies. The high prevalence and incidence of pressure ulcers in the sample analyzed reinforces the problem. It is suggested the use of the Braden Scale as assistance tool for preventing this type of preventable adverse event.
\end{abstract}

Keywords: Ulcers by pressure; Intensive Care Unit; Patient Safety; Nursing. 


\section{INTRODUÇÃO}

A segurança é o primeiro domínio da qualidade na assistência à saúde. Não há como oferecer uma boa assistência se esta não for feita com segurança. O conceito de Segurança do Paciente se refere à redução dos riscos de danos desnecessários associados à assistência em saúde até um mínimo aceitável. Esses danos desnecessários são conhecidos como eventos adversos. Assim, entende-se que os eventos adversos são danos não intencionais decorrentes da assistência prestada ao paciente, não relacionados à evolução natural da doença de base. Obrigatoriamente acarretam lesões mensuráveis nos pacientes afetados, óbito ou prolongamento do tempo de internação'.

A ocorrência de eventos adversos no ambiente da terapia intensiva infelizmente é uma rotina usual e frequente. Destaca-se que uma das consequências mais comuns resultantes de longa permanência em hospitais é o aparecimento de lesões de pele, especialmente as úlceras por pressão. A incidência aumenta proporcionalmente à combinação de fatores de risco, dentre eles idade avançada $e$ restrição no leito ${ }^{2}$.

As úlceras por pressão (UPP) constituem um dos principais eventos adversos encontrados em serviços e instituições de atenção à saúde. Para os pacientes, trazem dor e sofrimento e podem contribuir, em associação com outras causas, para a morte ${ }^{3}$.

Além disso, pelo importante impacto financeiro que representam às instituições de saúde, as UPP são consideradas problemas socioeconômicos e educacionais, devendo-se investir constantemente em ações de prevenção pensando na relação custo-benefício destas ações em relação às despesas de tratamento. Um paciente que desenvolve uma UPP demanda, em relação ao cuidado de enfermagem, $50 \%$ a mais de tempo, dado que evidencia a necessidade e relevância dos cuidados preventivos ${ }^{4}$.

A preocupação com a qualidade da assistência de enfermagem prestada ao paciente hospitalizado e em estado crítico é um desafio significativo para toda equipe que trabalha em Unidade de Terapia Intensiva (UTI). A prevenção de UPP deve ser uma prática criteriosa de qualidade, bem como uma ação prioritária. Para tanto, é 
Artigo Original

Atenção à Saúde

necessário lançar mão de estratégias que possam envolver a instituição e a equipe multidisciplinar que atua na UTI, dando prioridade ao enfermeiro que está diretamente e de forma contínua ligada a esse paciente.

O tema relativo à prevenção de úlceras por pressão já vem sendo estudado pelas autoras desde a participação em um projeto de extensão vinculado à disciplina de projetos de cuidado integral do $7^{\circ}$ semestre do curso de Enfermagem, quando se iniciou uma participação ativa em reuniões do Núcleo de Segurança do Paciente (NSP) de um hospital de referência, situado no município de Lages-SC. A partir disso, das demandas do hospital cenário e da parceria com o NSP, nasceu a proposta desse estudo. Tal proposta se delineou a partir de apontamento do problema, relativo ao tema segurança do paciente na prevenção de úlcera por pressão, observado durante atividades educativas realizadas junto à equipe de enfermagem da UTI. Sabendo que pacientes internados em UTI têm tendência a permanecerem muito tempo acamados, tendo risco de desenvolver UPP, buscou-se contribuir com a equipe de enfermagem desse setor, visando aprimorar a assistência de enfermagem na prevenção das UPPs, contribuindo para o bem-estar e qualidade de vida dos pacientes ali internados.

Posto isso, este trabalho teve como objetivo geral conhecer as ações assistenciais desenvolvidas pela equipe de enfermagem de uma Unidade de Terapia Intensiva de um hospital de referência da serra catarinense, em relação às UPPs.

\section{MÉTODO}

Estudo de caráter descritivo, com abordagem qualitativa, desenvolvido através do método de Pesquisa Convergente Assistencial (PCA), modalidade que busca integrar métodos de pesquisa relacionados com a prática assistencial ${ }^{5}$. A PCA estabelece a identificação de situações de pesquisa as quais surgem da prática de assistência dos profissionais, procurando definir potencialidades que possam auxiliar nas soluções adequadas para cada problema específico tanto na gestão, na assistência ou educação ${ }^{5}$. No presente estudo a implementação da PCA ocorreu por meio das fases de concepção, instrumentação, perscrutação, análise e interpretação.

A fase de concepção representou a escolha do tema, caracterizando o início do desenvolvimento do estudo, a partir de um projeto elaborado no $8^{\circ}$ semestre do curso de enfermagem, no ano de 2014, derivado da disciplina de Projetos de Cuidado 


\section{Atenção à Saúde}

Integral. Naquele momento foram realizadas capacitações com os profissionais de enfermagem do hospital cenário, abordando o tema segurança do paciente na prevenção de UPP. Durante esse período foi possível observar que o local onde há o maior risco para os pacientes desenvolverem UPP é em Unidade de Terapia Intensiva. A partir dessa observação, identificou-se a necessidade, em conjunto com o Núcleo de Segurança do Paciente, surgindo a proposta desse estudo.

O presente estudo foi desenvolvido no período de julho a novembro de 2015 e teve como cenário um hospital geral da serra catarinense. A pesquisa foi realizada em uma Unidade de Terapia Intensiva, que é composta por dez leitos. Os sujeitos do estudo foram os membros da equipe de enfermagem da UTI do hospital cenário, sendo dois enfermeiros e dez técnicos de enfermagem, totalizando 12 sujeitos. A identidade dos sujeitos foi preservada, sendo utilizados codinomes. Os preceitos éticos seguiram o disposto na Resolução 466/12 e o projeto foi aprovado pelo CEP da Universidade do Planalto Catarinense, sob o protocolo número 1.156.845.

A fase de perscrutação incluiu a coleta e o registro dos dados, sendo utilizado para tal a técnica de observação participante junto às equipes de enfermagem da UTI no turno da noite. Para o desenvolvimento das ações diárias de observação participante seguiu-se um roteiro, registrando-se a data da coleta de dados, o quantitativo de pacientes internados no dia, as ações preventivas realizadas junto aos pacientes com UPP e as notificações realizadas. Também foi utilizado o diário de campo, para os registros das ações diárias.

No primeiro encontro com a equipe de enfermagem houve a apresentação da proposta, por meio de abordagem individual, e a apresentação do Termo de Consentimento Livre e Esclarecido (TCLE). Também foi realizada uma atividade lúdica para a distribuição de codinomes aos sujeitos. No mesmo encontro foi questionado aos sujeitos sobre a problemática das UPP na UTI e os mesmos expressaram verbalmente suas ideias sobre a prevenção de UPPs, sendo registrado pelas autoras, em um cartaz, este conhecimento prévio dos sujeitos.

$\mathrm{Na}$ sequência das atividades de observação participante foi realizada a identificação dos pacientes da UTI em risco de desenvolver UPPs, junto à equipe de enfermagem, por meio de uma avaliação utilizando a escala de Braden. A escala de Braden é bastante utilizada nos Estados Unidos da América e foi publicada no ano de 
1987. No Brasil, a escala foi adaptada e validada no ano de 1992, e vem sendo aplicada em instituições de saúde 6 .

Esta escala deve ser preenchida somente pelo enfermeiro e auxilia na classificação do paciente quanto ao risco para desenvolvimento de UPPs. A escala estava disponível no prontuário do paciente pelo sistema eletrônico (Micromed) do hospital cenário. De acordo com a escala são avaliados seis fatores de risco, que são: (1) Percepção sensorial: referente à capacidade do cliente reagir significativamente ao desconforto relacionado à pressão; (2) Umidade: refere-se ao nível em que a pele é exposta à umidade; (3) Atividade: avalia o grau de atividade física; (4) Mobilidade: refere-se à capacidade do cliente em mudar e controlar a posição de seu corpo; (5) Nutrição: retrata o padrão usual de consumo alimentar do cliente; (6) Friç̧ão e Cisalhamento: retrata a dependência do cliente para a mobilização e posicionamento, contratura e agitação que podem levar à constante fricção?.

A aplicação da escala de Braden foi realizada pelas pesquisadoras, conjuntamente com a enfermeira do setor, em pacientes já internados na UTI. Nos horários em que as pesquisadoras não estavam presentes foi estimulado à equipe de enfermagem para fazê-lo.

Após classificação do risco para UPPs através da escala de Braden identificava-se o paciente através da colagem de adesivos coloridos no respectivo leito. A identificação por cores se deu considerando a seguinte classificação, elaborada pelas pesquisadoras: Sem risco: adesivo branco; Risco moderado: adesivo amarelo; Risco alto: adesivo vermelho.

Sobre a notificação da UPP, foi estimulada à equipe de enfermagem da UTI a fazê-la. Para tal, foi realizada uma abordagem em pequenos grupos, para reconhecimento do instrumento de notificação e para instrumentalização do seu preenchimento. Foi elaborado um painel comparativo referente à necessidade de notificação ou não de UPP. O painel foi organizado em duas colunas, uma intitulada "quando notificar UPP" e a outra "quando não notificar UPP". Para o desenvolvimento da atividade foi disponibilizado tarjetas com informações científicas sobre o assunto que contemplaram as duas colunas; cada sujeito pegou uma tarjeta e colou na coluna que achou indicado. Ao final da atividade foi feita uma discussão sobre a produção do grupo. Em outro momento, foi realizada uma atividade, por meio de dinâmica, para discutir e propor coletivamente estratégias relacionadas à prevenção de UPP. Os 
Artigo Original

Atenção à Saúde

sujeitos participantes demonstraram como é feito por eles as estratégias de prevenção de UPP e, após a demonstração, foi discutido coletivamente os métodos de cuidado adequados de se prevenir as UPPS.

\section{ANÁLISE E RESULTADOS}

A etapa de análise foi realizada a partir das informações contidas no diário de campo e dos dados registrados no instrumento de observação participante, ocorrendo de modo simultâneo, facilitando a identificação de dificuldades e lacunas ao longo do processo investigativo. Consideraram-se os registros referentes às abordagens educativas realizadas junto aos sujeitos, a aplicação da escala de Braden e as notificações de UPPs efetuadas, bem como todas as produções realizadas pelos 12 sujeitos ao longo do processo. Desta etapa, resultaram três categorias, sendo: (a) conhecimento profissional; (b) classificação de risco dos pacientes; (c) notificação das UPPs, as quais seguem.

\section{(a) Conhecimento profissional}

Nesta etapa refletiu-se junto aos sujeitos da pesquisa sobre a problemática das UPPs na UTI, sendo que os mesmos expressaram em palavras as suas ideias sobre a prevenção de UPP, sendo registradas em um cartaz. No decorrer da atividade foi possível registrar o conhecimento prévio dos sujeitos conforme as experiências profissionais iam sendo socializadas. Destacam-se algumas falas oriundas dessa etapa:

"O reposicionamento do paciente de duas e duas horas". (Tulipa)

"Coxins através de travesseiros nas regiões de atrito". (Hortênsia)

"Após o uso de colchão pneumático reduziu o índice de UPP na UTI'. (Margarida) 
"Importante na hora do banho do paciente sempre realizar a hidratação da pele". (Azaléia)

Foram também discutidas as ações preventivas para UPP usualmente realizadas pela equipe de enfermagem da UTI. Das formas de prevenção de UPP conhecidas e utilizadas pelos sujeitos, se destaca como a mais usual a mudança de decúbito, pois tem o beneficio de descomprimir áreas sob proeminências ósseas; em seguida aparece o colchão pneumático e o uso de hidratante. Ainda foram citados 0 uso de hidrocolóide, o ácido graxo, o óleo de girassol e os coxins.

\section{(b) Classificação de risco dos pacientes}

Nos encontros subsequentes iniciou-se junto aos sujeitos a classificação dos pacientes, sendo registrada no prontuário de cada paciente, juntamente com a enfermeira do setor. A classificação foi realizada pelo uso da escala de Braden. Outra estratégia foi a colagem de adesivos coloridos nos pés da cama de cada paciente internado na UTI, indicando o risco para desenvolvimento de UPP.

A utilização da escala de Braden pelos sujeitos foi considerada de fácil compreensão e aplicação, podendo ser usada durante o exame físico ou até mesmo na higiene corporal, facilitando assim a avaliação do paciente. A escala auxilia o enfermeiro na identificação de pacientes com risco de desenvolver úlceras, fazendo com que o mesmo realize um planejamento das ações de enfermagem para assim diminuir as chances de desenvolver UPP.

As avaliações semanais realizadas junto aos sujeitos foram produtivas, pois, nestes momentos, puderam-se discutir casos e oportunizou-se a declaração de que a escala de Braden é de fácil compreensão e aplicação, alegando que o uso deste instrumento não lhes toma tempo, conforme se destaca nos depoimentos a seguir:

"Não acho difícil, é fácil para aplicar e prática, não tenho nenhuma dificuldade seja de tempo ou compreensão na avaliação. A utilização da Escala de Braden pode se tornar um grande aliado do enfermeiro para aumentar a qualidade do serviço prestado ao paciente, pois ela permite conhecer o perfil 
do paciente e direciona sua sistematização de cuidado". (Hortênsia)

"Realizar a avaliação do paciente por meio da Escala de Braden, consequentemente promove ações que garantam a segurança do paciente durante sua internação hospitalar". (Cravo)

É importante ressaltar que se recomenda o uso da escala de Braden para auxiliar o profissional a identificar os pacientes de risco já no momento da admissão, e como no período de coleta de dados as pesquisadoras não acompanharam nenhuma admissão de pacientes, fez-se a classificação naqueles que já estavam internados na UTI.

Durante o período de coleta de dados foram classificados 17 pacientes, sendo que, destes, seis desenvolveram UPP. As regiões corporais onde as úlceras foram mais frequentes foram a do calcâneo e a sacral. O tempo médio de internação dos pacientes que desenvolveram úlcera foi de 18 a 30 dias e dos que não desenvolveram foi de 04 a 06 a seis dias. Destes 17 pacientes, 13 foram classificados em risco alto, $01 \mathrm{com}$ risco moderado e $03 \mathrm{com}$ risco baixo. Entre os escores de 18 a 21 para risco baixo e escores de 09 a 10 para riscos altos. Foram colados os adesivos coloridos em cada leito de acordo com o escore de classificação.

Considerando os fatores de risco para UPP, avaliados pela escala de Braden, encontrou-se que, em relação à percepção sensorial, as médias dos escores dos pacientes que desenvolveram úlceras foram menores do que os pacientes sem úlcera. Baixos subescores indicam que os pacientes que desenvolveram úlcera apresentavam-se entre "completamente limitado" e "muito limitado". O item atividade reflete o grau de atividade física do paciente. Observou-se que as médias dos subescores não apresentaram variações entre os pacientes com úlcera de pressão e sem úlcera, pois a maioria dos pacientes se apresentava constantemente acamados. Após a classificação através da escala de Braden, a partir de dados do próprio sistema eletrônico (Micromed) da referida escala, obtivermos as ações de enfermagem para a equipe realizar, que consiste em: 
Quadro1. Ações de Enfermagem pela Escala de Braden.

\begin{tabular}{|c|c|}
\hline \multicolumn{2}{|c|}{ Risco Baixo - Ações de Enfermagem } \\
\hline 1. & Manter a pele limpa e seca \\
\hline 2. & Hidratar a pele 01 vez ao dia após o banho \\
\hline 3. & Rouparia sem rugas \\
\hline 4. & Reposicionamento do paciente \\
\hline \multicolumn{2}{|c|}{ Risco Moderado - Ações de Enfermagem } \\
\hline 1. & Manter colchão piramidal \\
\hline 2. & Manter rouparia sem rugas \\
\hline 3. & Descomprimir proeminências ósseas (coxins, travesseiro) \\
\hline 4. & Realizar mudança de decúbito \\
\hline 5. & Hidratar a pele \\
\hline \multicolumn{2}{|c|}{ Risco Alto - Ações de Enfermagem } \\
\hline 1. & Manter colchão piramidal ou pneumático \\
\hline 2. & Manter rouparia sem rugas \\
\hline 3. & Descomprimir proeminências ósseas (coxins, travesseiro) \\
\hline 4. & Realizar mudança de decúbito \\
\hline $\begin{array}{l}5 . \\
\text { risco }\end{array}$ & Hidratar a pele + placa de hidrocolóide transparente nas regiões de maior \\
\hline 6. & Avaliação nutricional \\
\hline
\end{tabular}

Fonte: Micromed, 2015.

\section{(c) Notificação das UPPs}

Após a etapa de classificação dos pacientes pela escala de Braden, iniciouse o processo de orientação dos sujeitos a respeito da notificação de UPP, visando avaliar os resultados assistenciais e converter problemas potenciais em boas práticas.

O hospital cenário possui um formulário físico para notificação de queixa técnica ou evento adverso, onde todos os profissionais podem e devem notificar. Observou-se, no período do estudo, que muitos profissionais ainda não conheciam o formulário para notificação de eventos adversos.

Através do cartaz elaborado junto aos sujeitos foi possível notar o que cada profissional sabia sobre a notificação de úlceras por pressão. Ficou claro que, embora 
Artigo Original

Atenção à Saúde

técnicos e auxiliares de enfermagem entendam que podem notificar, revelam dúvida quanto à autorização para fazê-lo, bem como não se sentem esclarecidos sobre como proceder ao registro, preferindo relatar o fato ao enfermeiro para que este realize 0 registro. A partir dessa questão foi orientado sobre o processo de realização de notificações de UPP. Com base na abordagem utilizada, possibilitou-se compreender que o formulário de notificação de UPP, ou qualquer outro evento adverso, é instrumento fácil de ser preenchido e de fundamental auxílio à gestão da assistência ao paciente, permitindo aos profissionais prestar assistência adequada e estimular ações corretivas, com vistas à não repetição dos erros e prevenção de futuros erros.

Compreendeu-se que o preenchimento do instrumento de notificação traz também como benefício a organização de dados, possibilitando planejamento de ações preventivas de UPP para a equipe de enfermagem, pois muitos pacientes vêm de outro setor ou até mesmo de seu domicílio com UPP já instalada. O processo de notificação de UPP é um importante instrumento de gestão do cuidado para a enfermagem, pois permite avaliar os resultados assistenciais. Torna-se fundamental para identificar problemas potenciais e, posteriormente, adotar estratégias de intervenção, tendo em vista a promoção de boas práticas assistenciais.

\section{DISCUSSÃO}

A categoria relativa ao conhecimento dos profissionais frente à prevenção de UPP revelou que a mudança de decúbito é apontada como a principal ação a ser desenvolvida pela equipe de enfermagem na prevenção à UPP. Na sequência, foram elencadas outras ações de prevenção, como os métodos para prevenir o atrito cutâneo, a força de cisalhamento sobre a pele e a diminuição da pressão sobre as proeminências ósseas, como certifica o relato de Lírio:

"Mudança de decúbito, hidratação da pele, usando óleo de girassol, travesseiro entre as pernas". (Lírio)

A mudança de decúbito foi considerada por todos os sujeitos como o fator mais importante na prevenção de úlceras de pressão. Tal achado vem ao encontro do 
Artigo Original

Atenção à Saúde

que apontam outros estudos quando afirmam que $100 \%$ dos sujeitos utilizam a mudança de decúbito como medida preventiva à UPP8.

Com relação à frequência que é realizada a mudança de decúbito, os profissionais relataram que normalmente fazem essa mudança de 02 em 02 horas, como certificado na fala de Margarida:

"Geralmente faço de 02 em 02 horas conforme o relógio de mudança de decúbito”. (Margarida)

A mudança de decúbito a cada duas horas é uma das medidas preventivas utilizadas na Unidade de Terapia Intensiva. Este método evita o contato direto do cliente em superfície que diminui ou impede o fluxo sanguíneo tissular, durante um período prolongado, evitando a ocorrência da pressão no mesmo local e consequentemente evitando o desenvolvimento das úlceras por pressão ${ }^{9}$.

Os resultados demonstram ainda o conhecimento desses profissionais quanto à utilização de materiais e métodos para dar suporte à ação de mudar o paciente de posição, destacando o uso de coxins e da hidratação da pele com óleos, como descrito nas falas. Realizar o cuidado com a pele do paciente, como a limpeza, o uso de cremes hidratantes, a diminuição da umidade, a prevenção de massagem nas proeminências ósseas, o posicionamento adequado, a mudança de decúbito no horário correto, ainda, o cuidado com o transporte e a manipulação do paciente, são como formas de prevenir o surgimento da úlcera por pressão ${ }^{10}$.

Outro elemento de prevenção destacado pelos sujeitos foi o uso de colchão pneumático, sendo que referem que quando utilizado este colchão foi observada pela equipe de enfermagem a redução do índice de UPP. O uso de colchão próprio, tipo colchão de espuma, ar estático, ar dinâmico, gel ou água, redistribui o peso corporal, ocasiona a redução da pressão sob as proeminências ósseas à medida que o paciente afunda no fluído, propiciando uma superfície adicional que auxilia na sustentação do corpo, além da redução do peso corporal por unidade de área ${ }^{11}$.

A partir da análise dos resultados desse estudo pode-se observar que a UPP ainda é uma das grandes preocupações dos profissionais de enfermagem pelo fato de desempenharem o papel de cuidar dos indivíduos confiados a eles. No entanto é indispensável que estes profissionais tenham um instrumento para que possam 
identificar os pacientes com maior risco de desenvolver a úlcera e implementar medidas que visem reduzir o aparecimento da mesma.

Neste estudo, para classificação de risco de desenvolvimento de UPP, optouse pela utilização da escala de Braden, por ser um instrumento previamente validado e amplamente utilizado. Esta escala é utilizada pelos enfermeiros na avaliação dos riscos que os pacientes críticos são expostos para desenvolverem úlcera por pressão, permitindo que o profissional realize intervenções antes que o quadro de úlcera se instale". Destaca-se que, "como parte de protocolos de prevenção, as escalas de avaliação de risco de desenvolvimento de UP têm sido estudadas e implementadas em grupos de pacientes mais vulneráveis às alterações da integridade da pele" 6 . Apesar de estudos mostrarem a existência de mais de 40 escalas, somente seis têm sido testadas considerando sua validade preditiva, dentre elas, a escala de Braden ${ }^{6}$. A partir da utilização da escala de Braden para a classificação da UPP, pode-se identificar as ações de enfermagem a serem realizadas, conforme demonstrado no Quadro 1.

De modo geral, os resultados obtidos sugerem que essa escala deveria ser utilizada em todos os pacientes da UTI no momento da admissão e fazer a reavaliação do paciente todo dia, a fim de identificar aqueles com alto risco de desenvolver lesões por pressão e então realizar cuidados apropriados. Ainda, é possível afirmar que a escala de Braden é um instrumento que deveria ser utilizado em todos os pacientes durante a hospitalização, diminuindo assim o risco de formação de UPP.

Outro resultado importante foi a constatação de que, durante a classificação dos pacientes, dos seis que desenvolveram UPP, as regiões corporais mais frequentemente afetadas por úlceras foram a do calcâneo e a sacral. Fato este constatado também em outros estudos, destacando-se que

"no que concerne às regiões, constatou-se que o acometimento de UP na região sacral ocorreu em todos os pacientes, seguido pelo acometimento na região glútea, calcâneo, trocânter e medial do joelho. A maior incidência na região sacral e no calcâneo está associada aos maiores períodos em ventilação mecânica e permanência na UTI e, consequentemente, ao tempo prolongado de imobilização no leito, sugerindo que os pacientes mantidos em decúbito dorsal têm o risco aumentado para o desenvolvimento de UP."12 
Em relação às notificações da UPP, observou-se que as mesmas se mostraram como importante fonte de informação e alerta para a promoção da segurança do paciente. No ambiente hospitalar houve adesão da equipe à utilização do formulário, avaliada pelo aumento gradativo de notificações, estabelecendo um meio de comunicação prático de eventos adversos entre os profissionais de enfermagem e o NSP. Neste sentido, a gestão de risco é um processo no qual são criadas alternativas para diminuir ou eliminar os eventos adversos que podem ocorrer durante a prática dos profissionais da saúde. As instituições de saúde esforçam-se para desenvolver uma cultura de segurança do paciente e esperam que cada colaborador esteja preparado para identificar os potenciais perigos e para realizar as mudanças necessárias, ou seja, eliminação, redução e controle dos riscos ${ }^{13}$.

\section{CONSIDERAÇÕES FINAIS}

Por meio deste estudo foi possível identificar que a prevenção de UPP destaca-se como um cuidado importante na enfermagem. A identificação de risco e o uso das medidas preventivas recomendadas podem levar à redução da incidência de UPP, dificultar o seu agravamento, assim como favorecer a cicatrização das lesões presentes do paciente no hospital, melhorando a qualidade da assistência de enfermagem e do serviço de saúde.

Durante a coleta de dados, a PCA se caracterizou como um método muito eficaz para o desenvolvimento da pesquisa, associando assim a teoria e a pesquisa com a prática de assistência, viabilizando mudanças possíveis junto aos sujeitos.

Entretanto, para que a realidade presente nos serviços seja modificada e para que as recomendações sejam adotadas, torna-se necessário o engajamento dos profissionais de enfermagem em todos os níveis e situações da prática.

A utilização da Escala de Braden ajudou nas ações de cuidados com o paciente, que devem ser realizadas pela equipe de enfermagem. Nesta prática, os sujeitos foram orientados e estimulados a implantar a escala pelo sistema eletrônico. Tal prática era vista anteriormente como uma problemática e passou a ser vista como uma alternativa para a melhoria no cuidado com o paciente.

Considerando os objetivos iniciais do estudo evidencia-se que os dados encontrados assemelham-se à realidade descrita nos estudos existentes. A alta 
Artigo Original

Atenção à Saúde

prevalência e incidência de UPP na amostra analisada reforça o grave problema que as mesmas representam no contexto da assistência prestada nas UTIs.

Sobre as ações educativas realizadas, buscou-se contribuir na qualificação da equipe de enfermagem da UTI, visando colaborar na redução de complicações decorrentes das UPPs, bem como estimular o processo de notificação desses eventos adversos, promovendo a segurança do paciente. Destaca-se que, após a intervenção realizada, a UTI é atualmente um dos setores que mais notifica as UPPs no hospital cenário.

Finalmente, pode-se afirmar que este estudo sensibilizou os enfermeiros e suas equipes frente à necessidade de ações permanentes de estímulo à utilização de tecnologias assistenciais, neste caso para a prevenção das UPPs, por meio da implementação de um instrumento assistencial, que foi a Escala de Braden. Esta ação, em especial, trouxe consigo o reconhecimento da necessidade da prevenção para este tipo de evento adverso evitável.

\section{REFERÊNCIAS}

1. Anvisa. Assistência Segura: Uma reflexão Teórica Aplicada à Prática. $1^{\underline{a}}$ edição - [internet]. 2013. [acesso em28 abril 2015]. Disponível: <http://www20.anvisa.gov.br/segurancadopaciente/images/documentos/livros/Livro1Assistencia_Segura.pdf $>$.

2. Brasil, Ministério da Saúde. Gabinete do Ministro. Portaria ํㅜ 529 ,de $1^{\circ}=$ de abril de 2013. Institui o Programa Nacional de Segurança do Paciente (PNSP). [internet]. [acesso em28 abril 2015]. Disponível: <http://bvsms.saude.gov.br/bvs/saudelegis/gm/2013/prt0529_01_04_2013.html>.

3. Rede Brasileira de Enfermagem e Segurança do Paciente: Estratégias para a segurança do paciente: manual para profissionais da saúde / Rede Brasileira de Enfermagem e Segurança do Paciente. [internet]. Porto Alegre: EDIPUCRS, 2013. 132 p. [acesso em 25 de fev 2015]. Disponível: $<$ http://www.rebraensp.com.br/pdf/manual_seguranca_paciente.pdf>.

4. Ferreira JD, Aguira ES, Lima CLJ, Brito KKG, Costa MM, Soares MJG.O. Ações preventivas para úlcera por pressão em idosos com declínio funcional de mobilidade física no âmbito domiciliar. ESTIMA. 2016;14(1):36-42. [acesso em 01 de nov 2016]. Disponível: http://www.revistaestima.com.br/index.php/estima/article/view/118. 
5. Trentini M, Paim L. Pesquisa em enfermagem: uma modalidade convergente assistencial - Florianópolis: Ed da UFSC, 2004. 162p. - (Série enfermagem; REPENSUL).

6. Serpa LF, Santos VLCG, Campanili TCGF, Queiroz M. Validade preditiva da Escala de Braden para o risco de desenvolvimento de úlcera por pressão em pacientes críticos. Rev. Latino-Am. Enfermagem [Internet]. 2011[acesso em 03 de março 2015];19(1):[08 telas].Disponível: http://www.scielo.br/pdf/rlae/v19n1/pt_08.pdf

7. Souza CA, Santos I, Silva LD. Aplicando recomendações da Escala de Braden e prevenindo úlceras por pressão - evidências do cuidar em enfermagem. Revista Brasileira de Enfermagem. [internet]. 2006[acesso em 03de março 2015];59(3).Disponível:

http://www.scielo.br/scielo.php?script=sci_arttext\&pid=S0034716720060003006>.

8. Martins DA, Soares FFR. Conhecimento sobre prevenção e tratamento de úlceras de pressão entre trabalhadores de enfermagem em um hospital de Minas Gerais. Cogitare Enferm 2008;13(1):83-7. [acesso em 10 de março 2015]. Disponível: http://revistas.ufpr.br/cogitare/article/view/11956.

9. Fernandes DSO, Oitaven ATP. Assistência de enfermagem na prevenção da úlcera por pressão em pacientes internados em Unidade de Terapia Intensiva. Revisão de Literatura, 2011. [internet] [acesso em 12 de nov 2015]. Disponível: $<$ http://bibliotecaatualiza.com.br/arquivotcc/EU/EU19/FERNANDES-daniele-

OITAVENana.pdf>.

10. Ribeiro ARF. Úlcera de Pressão: revisão de conceitos. 2008. [internet] [acesso 12 de nov 2015]. Disponível: http://feridas.no.sapo.pt/ulceras_de_pressao.pdf.

11. Goulart FM et al. Prevenção de úlcera por pressão em pacientes acamados: uma revisão da literatura. Revista Objetiva [internet]. 2008. n. 8, 2008. [acesso em 12 de nov 2015].

<http://faculdadeobjetivo.com.br/arquivos/PrevencaoDeUlcera.pdf >.

12. Pedrosa IL, Silva MSML, Araújo AA. Schwanke CHA. DeCarli GA, Gomes I. Úlceras por pressão em idosos e não idosos: estudo de coorte histórica. Braz J Nurs [internet]. 2014 Mar [cited year month Day]; 13 (1):82-91 [acesso em 12 de nov 2015]. Disponível: http://www.objnursing.uff.br/index.php/nursing/article/view/4111/pdf_72.

13. Fassini $P$, Hahn GV. Riscos à segurança do paciente em Unidade de Internação Hospitalar: Concepções da Equipe de Enfermagem. Revista Enfermagem UFSM [internet]. 2012; 2(2): 290-299. [acesso em 02 de dez 2015]. Disponível: http://cascavel.cpd.ufsm.br/revistas/ojs-

2.2.2/index.php/reufsm/article/view/4966/3753. 